\title{
Le Paradoxe De La Croissance Universitaire
}

\author{
BRIGITTE SCHROEDER-GUDEHUS*
}

La recherche dont les résultats font l'objet de cet article a été entreprise à partir d'une double hypothèse: la première que les attitudes professionnelles et les modèles de comportement des scientifiques peuvent être façonnés aussi bien par le milieu au sein duquel ils reçurent leur formation supérieure que par leur pays d'origine; la seconde, que la distribution relative - parmi le personnel enseignant des universités - des plus hauts diplômes d'origine nationale et ceux d'origine étrangère peut, dans une certaine mesure, refléter l'autonomie potentielle d'une collectivité scientifique donnée.

En fait, la planification de l'output des universités - née de la préoccupation d'éviter des pénuries ou de déjouer la menace de produire des "chômeurs instruits" - est devenue un problème si crucial que la question de "canadianiser" les universités canadiennes est fréquemment évoquée en des termes puisés dans le vocabulaire des politiques d'immigration ou de main-d'oeuvre. Or, la question de savoir si oui ou non un professeur étranger prive d'emplois des "Canadiens méritoires"1 n'est qu'un aspect du problème de la "décanadianisation". L'autre aspect est d'ordre culturel et ne peut être dissocié du débat plus général qu'alimente la quête d'une identité collective et d'un idéal national. Dans cette perspective, les influences qui s'exercent - nonobstant les frontières nationales au sein de la collectivité scientifique internationale acquièrent des connotations politiques évidentes, bien qu'on hésite généralement à y appliquer des termes qui nous sont familiers dans l'univers politique: les termes de "domination" et de "dépendance", de "colonisation", de "soumission", ou d'"émancipation". On préfère recourir en effet aux notions plus souples de "centre" et de "périphérie", d'“autonomie" ou d'“adaptation" (adjustment $),{ }^{2}$ et on parle, par exemple, d'un "aiguillage" trop systématique vis-à-vis d'aspirations scientifiques étrangères pour désigner ce qu'on appellerait, dans un contexte purement politique, un "comportement de clientèle".

La sociologie des sciences est loin encore d'avoir éclairé de façon satisfaisante les conditions et les interactions qui affectent la position relative des pays au sein de la communauté scientifique internationale. De plus, une fois devenue d'intérêt publique, la ques-

*Professeur agrégé, Institut d'histoire et de sociopolitique des sciences, Université de Montréal.

1 "Worthy Canadians," Report of the Committee of Inquiry into Non-Canadian Influence in Alberta Post-secondary Education. Department of Advanced Education, Edmonton, Alberta, 1972, p. 28.

${ }^{2}$ Sur les notions de "centralité" et de "périphéralité", voir, par exemple, Joseph Ben-David, "Scientific Endeavor in Israel and the United States", The American Behavioral Scientist, VI, 4 (décembre 1962), pp. 12-16; Edward Shils, "Metropolis and Province in the Intellectual Community", in V.M. Dandekar, N. V. Sovani, Changing India, Bombay, 1961, pp. 275-294. 
tion de la portée politique éventuelle d'une "autonomie scientifique" ou, au contraire, d'une grande perméabilité du développement scientifique national vis-à-vis d'influences étrangères, tend à acquérir une dynamique qui lui est propre. Ainsi, avant même que ne soient explorées à fond les conditions dans lesquelles des communautés scientifiques données deviennent ou demeurent soit "centrales", soit "périphériques", l'analyse risque d'emprunter des voies suggérées par les articulations du débat politique. De plus, la politisation de la question risque d'affecter les conditions même de la recherche.

Ce phénomène se manifeste presque inévitablement quand la présence d'étrangers ou de citoyens nés à l'étranger est utilisée à titre d'indicateur d'influence étrangère (ou du degré de dépendance vis-à-vis de contributions étrangères). Il n'est qu'aggravé lorsque cette préoccupation d'une Ueberfremdung culturelle coincide avec un retrécissement du marché du travail. La focalisation sur la présence d'étrangers donne alors inévitablement aux discussions un parfum de "nativism" ou même de xénophobie, suscitant par là même un climat de méfiance, peu propice à la recherche. C'est le cas au Canada, où certaines universités refusèrent, il y a quelque temps, de divulguer toute information sur la citoyenneté ou le lieu de naissance de leur personnel enseignant ${ }^{3}$. Dans ces circonstances, il est peu probable que la disponibilité des données ne s'améliore et qu'à court terme le débat entourant les affiliations nationales du personnel enseignant ne devienne moins délicat. Se trouve-t-il, de fait, quelque substitut valable? La tendance, toute récente ${ }^{4}$, d'accorder au pays d'origine du dernier diplôme une attention au moins égale à celle qu'on accordait habituellement à la citoyenneté ou au pays de naissance, nous semble révélatrice à cet égard.

Il importe de souligner ici que le problème des influences étrangères qui se manifestent dans la vie intellectuelle et scientifique canadienne et que d'aucuns jugent excessives, ne peut être envisagé sans qu'on le place sur l'arrière-plan de l'expansion de l'enseignement post-secondaire que le pays a connue après la deuxième guerre mondiale. Au cours de cette période, les universités canadiennes se sont vues investies de la mission d'importer des compétences, ce qu'elles firent, soit en important des ressortissants étrangers, soit en "ré-important" des Canadiens qui avaient acquis ces compétences dans des universités étrangères. Or, - comme nous l'avons souligné en présentant notre première hypothèse des aspirations, des méthodes et des styles étrangers ne s'implantent pas uniquement par la présence d'enseignants étrangers: il y a de fortes probabilités que des particularites non-nationales soient "rapatriées" par des natifs qui reçoivent une partie significative de leur formation à l'extérieur.

L'objectif de notre recherche n'était pas de vérifier cette hypothèse; il se situait à un niveau plus modeste et ne visait qu'á dégager rapport diplômes d'origine nationale / d'origine étrangère, tel qu'il évolua au cours des années 1950 à 1972 . La méthode employée appelle quelques réserves. Considérer comme équivalents le milieu national où un scientifique reçut la part la plus significative de sa formation et le pays où il obtint

3“28.4 pour cent des professeurs de Laval sont d'origine étrangère", Le Soleil, du 28 novembre 1972. Voir aussi l'expérience du Moir Committee, dont fait état le Report of the Committee of Inquiry into Non-Canadian Influence, op. cit., pp. 3-7.

${ }^{4}$ Elle se manifeste dans le Interim Report of the Select Committee on Economic and Cultural Nationalism. Colleges and Universities in Ontario. Toronto, 1973, pp. 7-9, 97-123. 
son plus haut diplôme ne tient évidemment pas compte d'une formation acquise sans qu'elle ne soit sanctionnée par un grade universitaire (comme, par exemple, les stages post-doctoraux). Aucune distinction ne fut d'ailleurs faite entre les doctorats, les maitrises et les baccalauréats ${ }^{5}$, l'influence qu'a le milieu de formation n'étant pas nécessairement fonction du niveau du diplôme obtenu. Nous avons organisé nos données en distinguant certaines grandes catégories disciplinaires (sciences exactes, ingéniérie, sciences sociales) ${ }^{6}$, les grandes régions canadiennes (Ouest, Ontario, Québec anglophone ${ }^{7}$, Québec francophone $^{8}$, Maritimes), l'affiliation des diplômés (universités, Conseil National de Recherches), et les principaux pays "émetteurs" de diplômes (Canada, U.S.A., Royaume-Uni, France, pays du Commonwealth).

En vingt-deux ans, la part des professeurs ayant obtenu leur dernier diplôme dans des universités canadiennes tomba d'une moyenne de $64.9 \%$ en 1950 à une moyenne de $40.3 \%$ en 1972. Le taux de déclin ne fut pas tout à fait régulier sur l'ensemble de la période, ni tout à fait similaire pour toutes les disciplines. Après une accélération remarquable au cours des années 1960, un certain plafonnement s'annonce depuis 1970 (fig. 1). On remarque une évidente - bien que non étonnante - coincidence entre le déclin de la part de diplômes canadiens et le rythme d'expansion du personnel universitaire enseignant (fig. 2).

${ }^{5}$ La distribution par niveau du diplôme (Ph.D., Maîtrise, Baccalauréat) en physique et en chimie révèle que la part relative de diplômes obtenus à l'étranger coincide avec un changement qualitatif de la composition du corps professoral: entre 1950 et 1972 la proportion de professeurs détenant un doctorat n'a cessé d'augmenter (par exemple, en physique: $49.2 \%$ en 1950, 79.7\% en 1972; en chimie: $61.8 \%$ en $1950 ; 87.9 \%$ en 1972). Le Québec francophone se signale d'ailleurs à la fois par la proportion la plus élevée de diplômes canadiens et la porportion la plus faible de professeurs détenteurs de doctorats, bien que l'écart par rapport à la moyenne nationale tende à se réduire rapidement.

6Sources: Commonwealth Universities Yearbook, 1950, 1956, 1962, 1967, 1970, 1972. Conseil National de Recherches du Canada, Annual Review, 1950, 1956, 1962, 1967, 1970, 1972. Statistique Canada, Survey of Higher Education. Catalogue annuel 81-204/211. Méthode: on n'a pas procédé à un échantillonnage. Les enseignants à plein temps ont été recensés de manière exhaustive, selon leur affiliation disciplinaire (le département auquel ils sont rattachés) et le pays où ils ont obtenu leur dernier diplôme.

Dans un premier recensement, d'autres disciplines avaient été inclues, telles les humanités, l'éducation, les sciences de la vie et la médecine. Elles ont été éliminées par la suite afin de maintenir le projet dans des limites raisonnables. Comme on pouvait s'y attendre, l'agriculture, l'éducation, les sciences de la vie et la médecine montrèrent un comportement proche de celui de l'ingénierie, c'est-à-dire une proportion relativement élevée, quoique décroissante, de diplômes canadiens. Les humanités ressemblaient à cet égard aux sciences sociales.

Dans deux secteurs non-universitaires, le recensement de la distribution des derniers diplômes obtenus par pays d'origine a donné les résultats suivants (1973):

Chercheurs employés par le gouvernment fédéral: Canada (38.4); Etats-Unis (25.3); Royaume-Uni

(9.5); France (0.4); Commonwealth (1.5); autres (4.9); inconnus (20.1); $\mathrm{N}=1964$.

Chercheurs employés par une grande entreprise industrielle: Canada (77.5); Etats-Unis (7.9); Royaume-Uni (11.2); France (1.1); autres (2.2); $\mathrm{N}=89$.

${ }^{7}$ McGill, Sir George Williams, Bishop.

${ }^{8}$ Laval, Université de Montréal, Université de Sherbrooke, Université du Québec. 


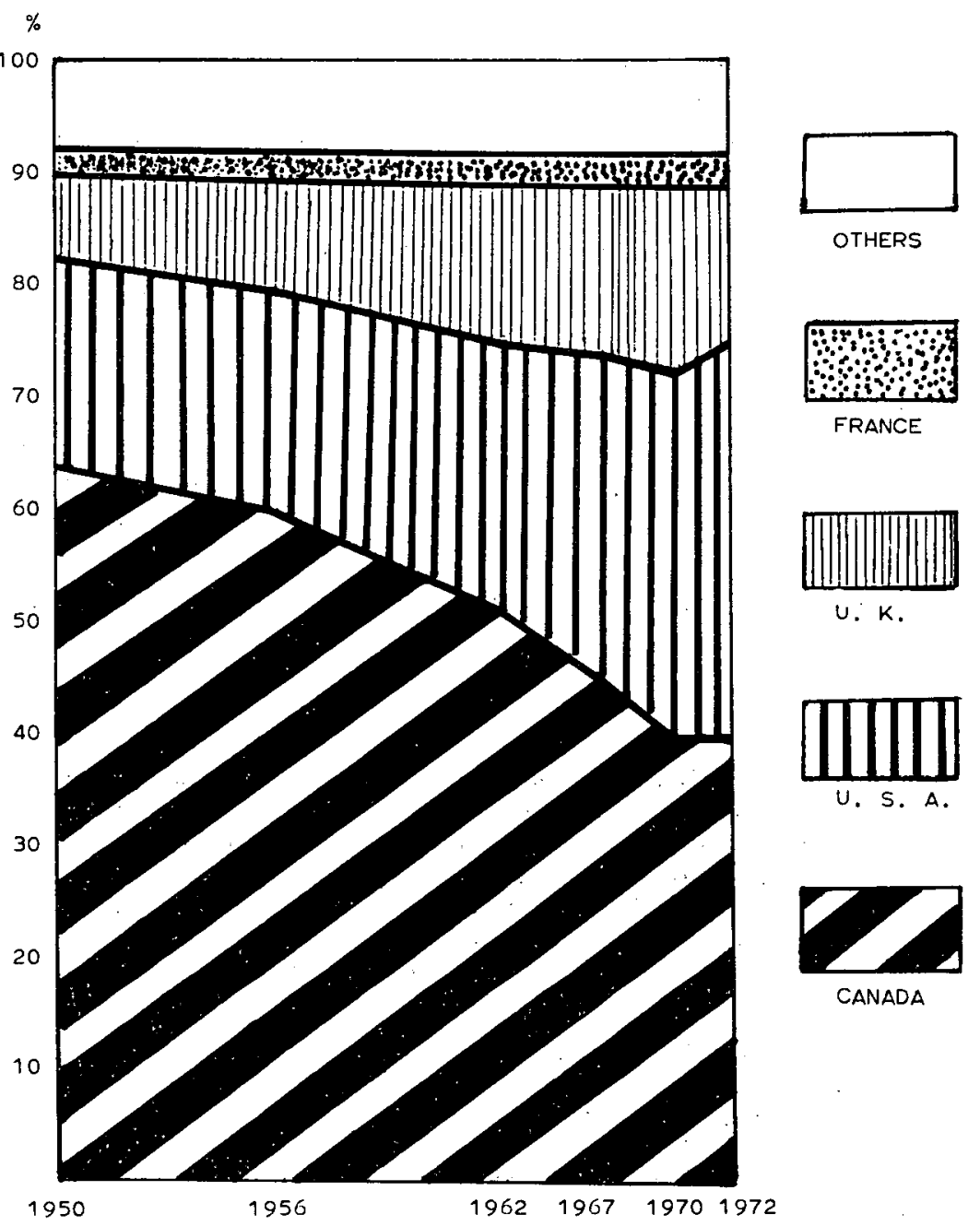

Figure 1. Pourcentage de professeurs détenant des diplômes canadiens, américains, britanniques, français et autres (toutes disciplines recensées), 1950-1972.

Distribution par discipline

Bien que le déclin ait été général, il a varié d'intensité d'une discipline à l'autre: parmi les disciplines recensées, l'ingénierie garde constamment la plus grande proportion de diplômes canadiens, se situant considérablement au-dessus de la moyenne nationale jusqu'au milieu des années 1960. Alors que dans les départements d'ingénierie les diplômes canadiens tombérent de $72.5 \%$ en 1950 à $44.7 \%$ en 1972 , le déclin fut moins accentué dans les sciences exactes (60.9\% en 1950 à $41.8 \%$ en 1972). Mais c'est sans doute dans les sciences sociales que la "dé-canadianisation" est la plus accentuée: tombant de $62 \%$ en 1950 à $37.2 \%$ en 1972 , le pourcentage du personnel universitaire enseignant 


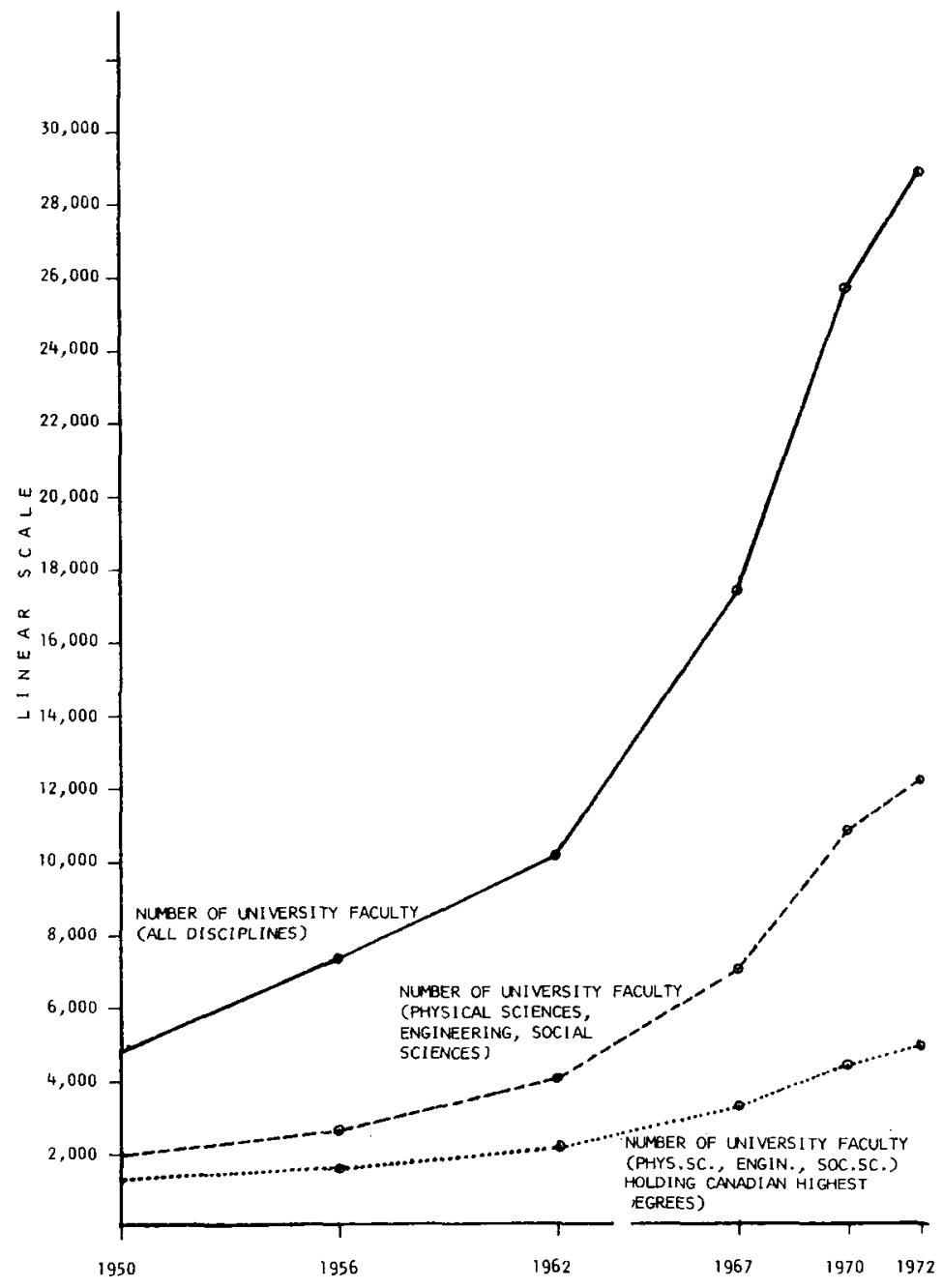

Figure 2. Personnel enseignant des universités canadiennes. Distribution par origine du diplôme, 1950-1972.

détenant un dernier diplôme canadien se trouva toujours, depuis le milieu des années 1960, au-dessous de la moyenne nationale. A l'heure actuelle, les disparités de comportement tendent à diminuer: la proportion de diplômes dans toutes les disciplines recensées semble se stabiliser autour de $40 \%$, les déviations se situant entre $+4.4 \%$ (ingénierie) et $-3: 1 \%$ (sciences sociales) (fig. 3).

\section{Distribution par région}

Ce sont le Québec et les provinces de l'Ouest qui manifestent des comportements les plus spécifiques (fig. 4). Parmi toutes les régions, la province de Québec est la moins "dé- 
14 Brigitte Schroeder-Gudehus

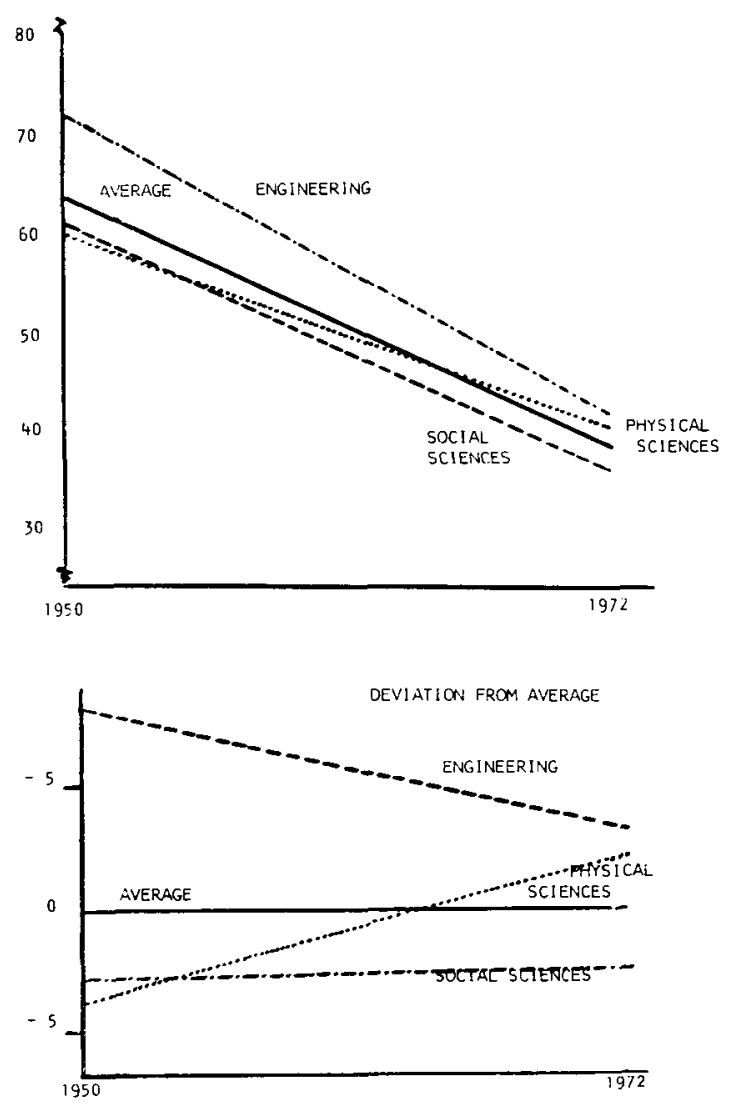

Figure 3. Pourcentage du personnel enseignant détenant des diplômes canadiens 19501972.

canadianisée", et ceci tant pour les universités anglophones que francophones: en 1972, $54.8 \%$ des professeurs des universités québecoises détiennent un dernier diplôme canadien, alors que la moyenne nationale se situe à $40.3 \%$. Malgré un déclin de $74.2 \%$ en 1950 à $58.4 \%$ en 1972, les universités francophones se maintiennent constamment à un niveau supérieur de 8 à $15 \%$ à la moyenne nationale. Les universités anglophones se distinguent par une remarquable stabilité: de 1950 à 1972, la part des diplômes canadiens n'a décru que de $6.1 \%$.

Dans les provinces de l'Ouest, les détenteurs de derniers diplômes étrangers sont nettement les plus nombreux. Du milieu des années 1950, jusqu'à 1972, moins de la moitié des professeurs détenaient des diplômes canadiens. Avec un déclin de $28.9 \%$ pendant la période étudiée (de 59.3 en 1950 à 30.4 en 1972), ce sont ces provinces dont les universités présentent le plus haut degré de "dé-canadianisation".

Un relevé par région et par discipline révèle une image encore plus 'significative: la part des derniers diplômes canadiens atteint son plus haut pourcentage avec les sciences 


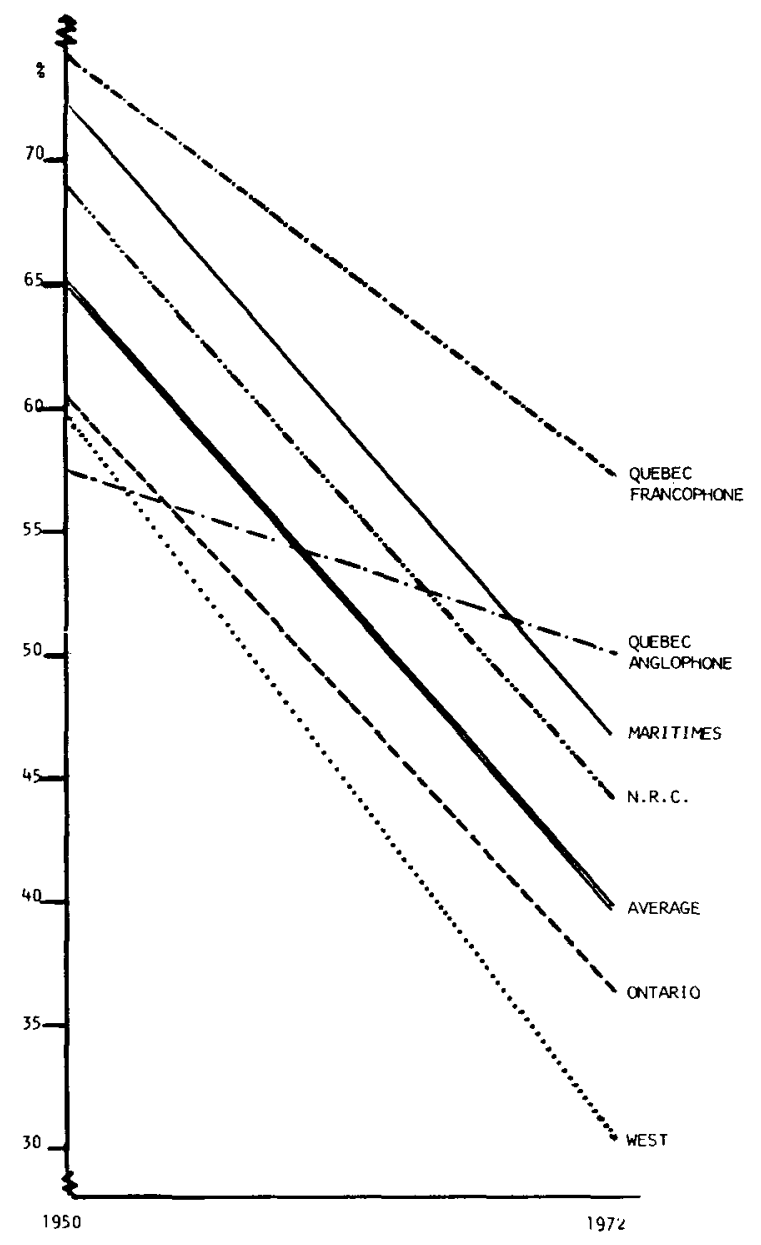

Figure 4. Pourcentage de derniers diplômes canadiens par région (toutes disciplines recensées), 1950-1972.

exactes dans les universités francophones (60\% en 1972) et descend à son niveau le plus bas avec les sciences sociales dans les universités des provinces de l'Ouest (26.9\% en 1972).

\section{Diplômes étrangers: distribution par pays d'origine}

La distribution des diplômes étrangers dans les disciplines recensées, confirmerait-elle ce que d'aucuns ont appellé 1' "américanisation massive" de la science canadienne ${ }^{9}$ ? C'est dans les provinces de l'ouest que l'on trouve la proportion la plus élevée de derniers diplômes américains dans toutes les disciplines recensées. Dans les universités franco-

${ }^{9}$ L. Trainor, "The Americanization of Canadian Science: How We Lose By Default," Science Forum, III, 2 (avril 1970), p. 8. 
phones du Québec par contre, le pourcentage de derniers diplômes américains excède à peine celui des diplômes français (Etats-Unis: 15.3\%, France: 11.8\%). Le Conseil National de Recherches se signale par une très forte proportion de diplômes britanniques ( $22.4 \%$ contre une moyenne nationale de $14.4 \%$, en 1972 ; Etats-Unis: $13.8 \%$, contre une moyenne nationale de $34.5 \%$ ).

Dans l'ensemble, toutes régions et toutes disciplines réunies, le pourcentage de derniers diplômes américains semble toujours légèrement en progression, cet accroissement étant dû particulièrement aux sciences sociales, la distribution dans les autres disciplines demeurant remarquablement stable (fig. 5) ${ }^{10}$. En fait, parmi les disciplınes recensées, ce sont les sciences sociales qui ont le plus largement fait appel à des enseignants ayant reçu leur dernier diplôme dans une université américaine, exception faite des universités francophones du Québec, où ce sont les diplômés français qui se retrouvent en plus grand nombre. Dans les sciences exactes et l'ingénirie, la proportion de derniers diplômes britanniques est relativement élevée parmi les diplômes non-canadiens, plus particulièrement, comme nous l'avons déjà signalé, au Conseil National de Recherches.

Le C.N.R. et le Québec ont ceci de particulier que les diplômes présentent une plus grande diversité d'origine: les pays "autres" que les Etats-Unis, le Royaume-Uni ou la France y apparaissent plus fréquemment qu'ailleurs. Cette diversité des origines de derniers diplômes ressort plus particulièrement du recensement des diplômes que détiennent les chercheurs du C.N.R. dans les domaines de la physique et de la chimie.

Il n'y a rien dans ces résultats qui ne soit impossible d'expliquer ou qui ne soit surprenant, si ce n'est l'intensité et la régularité du déclin de la part relative de derniers diplômes canadiens.

Une comparaison rapide entre la distribution du personnel universitaire enseignant par citoyenneté et par pays du dernier diplôme démontre que ces deux indicateurs ne sont pas interchangeables: chacun reflète un aspect différent d'un même probléme, problème qui est celui de l'effet que l'un comme l'autre de ces deux phénomènes risquent d'avoir sur des communautés scientifiques émergentes (fig 6). En fait, le recours à la distribution par origine du dernier diplôme obtenu semble, à certains égards, préférable. Non seulement les données sont-elles plus aisément disponibles, mais encore l'origine du dernier diplôme est-elle sans doute un indicateur plus significatif, quánd il s'agit de cerner des réseaux privilégiés de communication, de cerner l'origine d'éventuels emprunts conceptuels ou méthodologiques: en d'autres termes, d'identifier des tendances potentielles d'“adaptation" ou d" "émancipation".

Ce bref article avait pour objectif de présenter des résultats d'une première exploration et de suggérer quelques avenues de recherche; il ne prétend pas fournir des interprétations, ni se prononcer avec autorité sur la validité de cet indicateur. Il serait important d'explorer davantage le lien éventuel qui existe entre le rapport diplômes d'origine nationale/d'origine étrangère et d'autres indicateurs de "centralité" et de "périphéralité": concepts qui sont d'ailleurs loin d'être définis avec précision.

${ }^{10}$ De fortes oscillations de pourcentages, plus particulièrement au cours de la première décennie, doivent être interprétées avec prudence, du fait que les nombres absolus sont souvent peu élevés. 


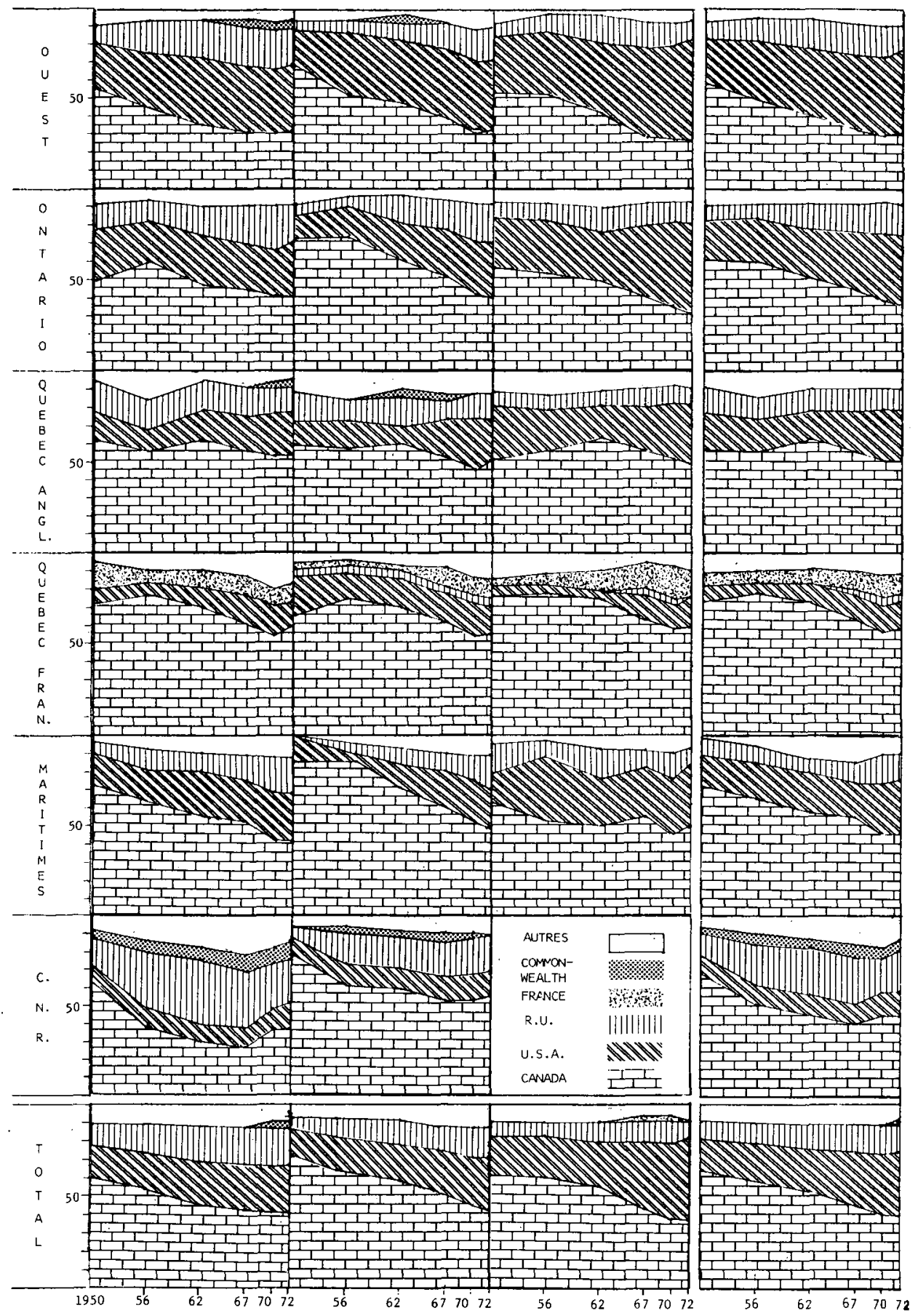

Figure 5. Personnel enseignant des universités canadiennes. Distribution par disciplines recencées, région et pays d'origine, 1950-1972. 

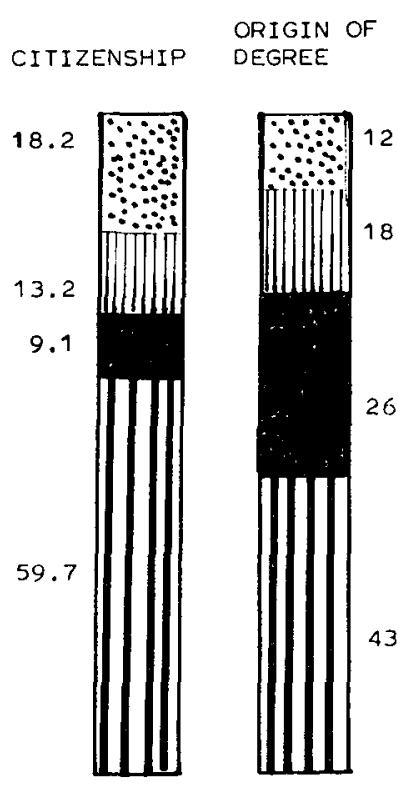

$N=5070$

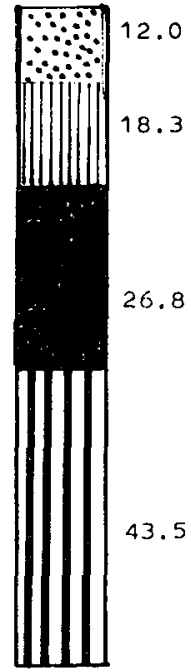

$N=6225$
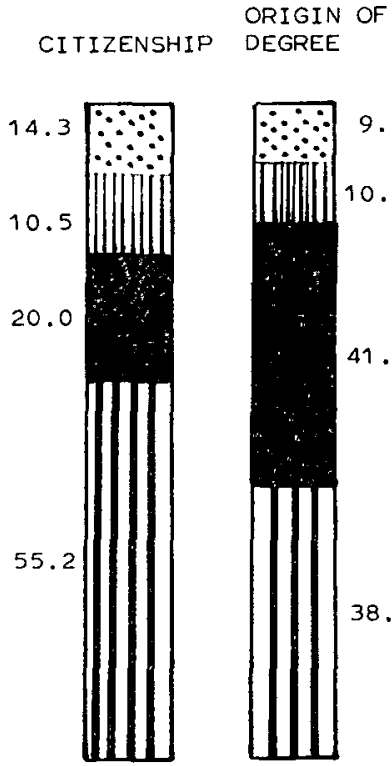

$N=5604$

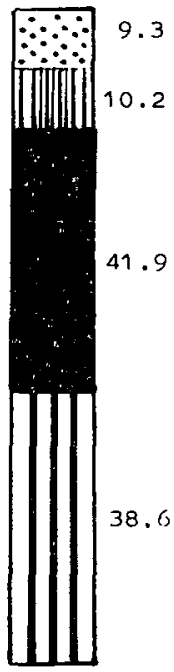

$N=5358$

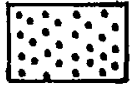

OTHER

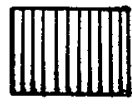

U. K.

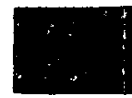

U. S. A.

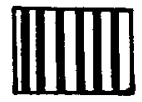

CANADA

$1970-71$

Figure 6. Personnel universitaire enseignant par citoyenneté et par pays d'origine du dernier diplôme (1970-71) 11 .

La diponibilité de tels indicateurs nous permettrait, en effet, d'attaquer avec une plus grande chance de succès, certains problèmes que posent les politiques de l'enseignement supèrieur. Car, s'il est utile de savoir ce qu'est le rapport, il serait certainement plus utile encore de savoir ce que le rapport entre diplômes d'origine nationale et ceux d'origine étrangère devrait être, et comment on pourrait l'infléchir. Il ne peut s'agir, bien entendu, de plaider pour une politique qui maintiendrait le recrutement de professeurs et la formation de troisième cycle aussi près que possible du niveau autarcique. Il risque d'y avoir un seuil, cependant, à partir duquel la participation aux activités scientifiques de niveau avancé d'un autre pays comporte autant de risques que d'avantages. Il y a risque, en effet, qu'une communauté scientifique émergente importe et assimile ainsi, sans examen critique, les normes et les méthodes d'un pays étranger, et qu'elle articule inconsciemment ses aspirations et ses objectifs sur les préoccupations de puissances scientifiques dominantes. En ce qui concerne les sciences sociales, cette assimilation implique sans doute plus que les objectifs et les normes de la discipline.

${ }^{11}$ Source pour les données concernant la citoyenneté du personnel universitaire enseignant: M. von Zur-Muehlen, "The Ph.D. dilemma in Canada," in Canadian Higher Education in the Seventies, Conseil Economique du Canada, Ottawa, 1972, plus particulièrement pp. 92-101. 
Table 1. Pays d'origine du dernier diplome obtenu (toutes disciplines recensées), 19501972.

\begin{tabular}{lcccccr}
\hline CANADA & 1950 & 1956 & 1962 & 1967 & 1970 & 1972 \\
\hline CANADA & 64.9 & 59.7 & 53.7 & 45.9 & 40.6 & 40.3 \\
\hline U.S.A. & 16.9 & 19.7 & 21.8 & 28.2 & 32.9 & 34.5 \\
\hline ROYAUME-UNI & 8.8 & 11.0 & 14.4 & 15.1 & 15.5 & 14.4 \\
\hline FRANCE & 1.6 & 1.7 & 1.8 & 2.2 & 2.2 & 2.5 \\
\hline COMMONWEALTH & 1.1 & 1.2 & 1.8 & 2.1 & 2.5 & 2.1 \\
\hline AUTRES & 6.7 & 6.7 & 6.5 & 6.5 & 6.3 & 6.2 \\
\hline N & 2089 & 2670 & 4168 & 7317 & 10996 & 12441 \\
\hline
\end{tabular}

Il ne fait aucun doute que le cas du Canada est particulier. Il est bon de se rappeler, en fait, que le pouvoir d'attraction exercé par les Etats-Unis ne tient pas uniquement au prestige de ses institutions universitaires. ${ }^{12}$ Ceux qui, au Canada, se préoccupent de l'étude des facteurs d'une "intégration a-symétrique" à la science américaine insistent généralement davantage sur la mainmise étrangère sur les industries à forte composante scientifique que sur le profil de formation de la gente scientifique canadienne. Le développement que connut le Canada dans le domaine de la recherche scientifique et de l'enseignement supérieur, a sans aucun doute été tributaire de la proximité géographique et linguistique des Etats-Unis. Ce fait se reflète nécessairement dans le développement du rapport entre diplômes d'origine nationale et diplômes d'origine étrangère. Cependant il importe maintenant de savoir si ce développement est comparable à ce qui se produisit ou se produit dans d'autres pays subissant des transformations du même type. ${ }^{13}$

12 Voir, par exemple, Ronald M. Pavalko, "Talent migration: Canadian students in the United States," International Review of Education, XIV, 3 (1968), pp. 300-322; Yochanan Comay, "The benefits and costs of study abroad and migration," Canadian Journal of Economics, III, 2 (mai 1970), pp. 300-308; "The migration of professionals; an empirical analysis," ibid., V, 3 (août 1972), pp. 419 429.

${ }^{13}$ Voir par exemple, en ce qui concerne l'Inde, les études récentes de Thomas Eisemon, U.S.Educated Engineering Faculty. Bombay, 1974; "Educational Transfer: the Implications of Foreign Educational Assistance", Interchange, 1975 (sous presse); Michael J. Moravcsik, "The transmission of a Scientific Civilization", Bulletin of the Atomic Scientists, XXIX (3), Mars 1973, pp. 25-28. 
Il existe, bien sûr, d'un pays à l'autre, des différences dans les modes de recrutement du personnel enseignant, différences de tradition, de contraintes légales, dont il faut tenir compte. En fait, le type d'analyse que nous présentons ici ne constitue qu'un pas somme toute modeste vers une compréhension plus adéquate de l'impact qu'une importation massive de compétences acquises à l'étranger peut avoir sur l'évolution des collectivités scientifiques nationales et le contenu de leurs activités. Jusqu'ici, la recherche s'est surtout intéressée à ce phénomène quand il s'agissait d'en étudier les conséquences dans les secteurs des humanités et des sciences sociales, - sans doute parce que les problèmes qu'il y crée sont plus immédiatement perceptibles que dans le domaine des sciences exactes. Il est néanmoins nécessaire d'étendre l'étude à toutes les branches de l'entreprise scientifique si l'on veut saisir de façon plus différenciée l'impact des importations de compétences, impact dont la nature, l'intensité et les effets à long terme varient sans doute en fonction des disciplines et du contexte sociopolitique et culturel dans lequel s'insère ce type particulier de transfert de connaissances.

L'auteur tient á remercier Derek de Solla Price qui, par l'intérêt qu 'il y porta et les conseils qu'il prodigua, contribua largement à cette recherche. Louise Dandurand apporta une assistance précieuse en ce qui concerne la collecte et l'analyse des données. 